\title{
Latest status of PMTs and related sensors
}

\author{
Motohiro Suyama ${ }^{1}$ \\ Electron Tube Division, Hamamatsu Photonics K.K. \\ 314-5 Shimokanzo, Iwata, Japan \\ E-mail: suyama@etd.hpk.co.jp
}

Photomultiplier tubes (PMTs), which incorporate a photocathode and a dynode chain for electron multiplication in vacuum, are featured by high gain to detect a single photon and fast response time of nano second with large effective area up to 20 inches. Single-photon detection with such large effective area at low dark count rate of a few thousands $\mathrm{Hz}$ or less cannot be achieved by any other devices. Therefore, PMTs are still useful for various applications, such as biology, medical, chemistry, industry as well as high-energy physics experiments. Varieties of PMTs in effective area, photocathode material and electron multiplication mechanisms are available for individual purpose, furthermore, the PMTs are still evolving to satisfy the requirements from advanced frontier science, such as high-energy physics.

One of the recent achievements is a big jump of photocathode sensitivity. Due to careful control of the photocathode process, the peak quantum efficiency of bialkali photocathode (ultra bialkali, UBA) reaches $43 \%$, for example for R7600, quite high compared to $27 \%$ of conventional ones. Another challenge has been done on electron-multiplication stage to realize a hybrid photo-detector (HPD). Instead of the dynode chain, an avalanche diode is incorporated in a tube to receive and multiply electrons. A high-speed HPD has just been developed by adjusting transit time of electrons from the photocathode and the avalanche diode, as well as using a small avalanche diode for fast time response. This HPD shows a single-photon timing resolution of $50 \mathrm{ps}$ in FWHM and a high gain of $10^{5}$. In this article, recent advancements of PMTs and related sensors will be described.

International workshop on new photon-detectors PD07

Kobe University, Kobe, Japan

27-29 June, 200

\footnotetext{
${ }^{1}$ Motohiro Suyama
} 


\section{Introduction}

Photomultiplier tubes (PMTs), which incorporate a photocathode and a dynode chain for electron multiplication in vacuum, are featured by high gain to detect a single photon and fast response time of nano second with large effective area up to 20 inches. Single-photon detection with such large effective area at low dark count rate of a few thousands $\mathrm{Hz}$ or less cannot be achieved by any other devices. Therefore, PMTs are still useful for various applications, such as biology, medical, chemistry, industry as well as high-energy physics experiments. Varieties of PMTs in effective area, photocathode material and electron multiplication mechanisms are available for individual purpose, furthermore, the PMTs are still evolving to satisfy the requirements from advanced frontier science, such as high-energy physics. In this article, recent advancements of PMTs and related sensors will be shown.

\section{PMTs}

In this section, recent advancements of PMTs are shown, as well as operational principle and general features.

\subsection{Structure and common features of PMTs}

A sectional drawing of typical PMT is shown in Figure 1 [1]. PMT is a vacuum tube incorporating a photocathode to emit electrons in response to incident photons and a dynode chain to multiply electrons by a factor of $10^{6}$. Since a photocathode is made by a reaction of alkali vapor, such as potassium and/or cesium, large size, such as $8,10,13$ or 20 inches are available. Dark current from the photocathode is 0.01 to $0.1 \mathrm{~Hz} / \mathrm{mm}^{2}$ for bialkali photocathode at room temperature; very low comparing with semiconductor devices sensitive to single photons. Response time of the photocathode is less than pico second [2] due to small thickness of several tens nm.

A dynode chain is a series of electrodes called dynode. Each dynode multiplies electrons by a factor of 5 , for example, as the secondary electron multiplication. With 8 or 10 dynodes, electrons are multiplied by a factor of $10^{6}$ or higher and are output from the anode. A transit time spread of electrons through a dynode chain deteriorates timing resolution of incident photon by an order of nano second or less; acceptable for most of applications. Thanks to this high gain and high timing resolution, detection of single photon is easily achieved at timing resolution of less than nano second.

In the case of large format PMT, an electro-static focusing lens in vacuum is used to combine a photocathode of large area and a dynode chain. Electrons from the photocathode are focused on a much smaller dynode, as shown in Figure 2. This kind of flexibility on electric field in vacuum tube makes it possible for PMTs to be more useful and more finely tuned for each application.

Contrary to these versatilities, PMTs still have rooms to be improved. The quantum efficiency of normal photocathode has been 20 to $30 \%$. Thus, only a part of photons can be converted to electrons at the photocathode. The dynode chain is bulky, comparing with semiconductor devices. Another disadvantage of the conventional PMTs is that they cannot be operated in a magnetic field, because electron trajectory is seriously distorted by the field. In the following sections, recently developed photocathode with higher quantum efficiency is 
introduced. A flat panel PMT using a low-profile dynode is shown as well. A PMT using microchannel plates instead of the dynode chain is discussed in terms of better timing resolution and applicability in a high magnetic field.

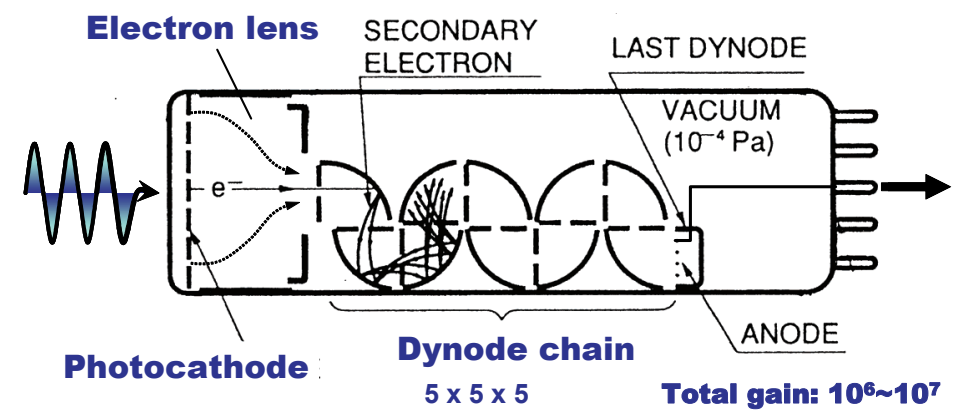

Figure 1: Sectional drawing of a typical PMT, which incorporates a photocathode and a dynode chain in a vacuum envelope.

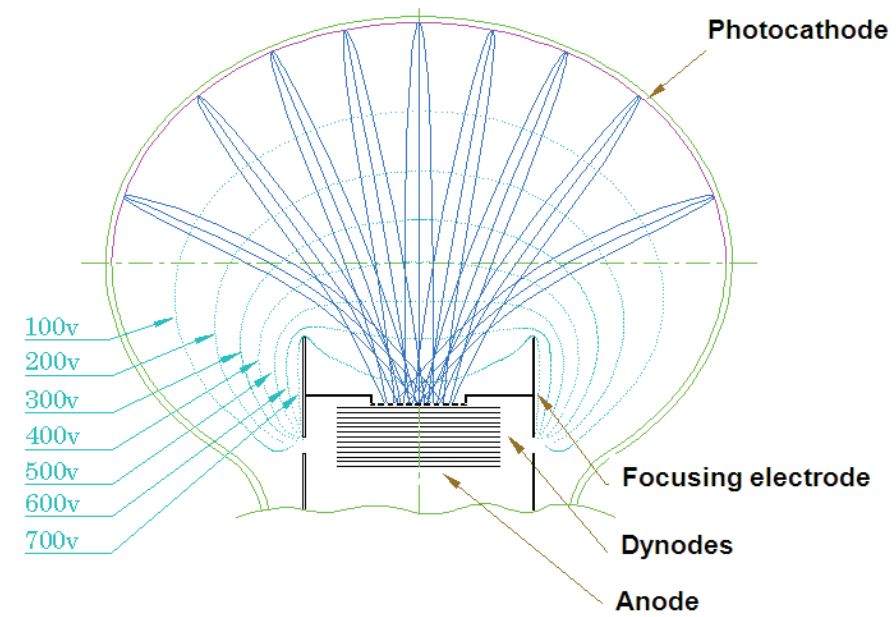

Figure 2: Electrons from the photocathode of large format PMT are focused on the dynode by electrostatic focusing lens. Electron trajectory, as well as equi-potential, is shown.

\subsection{Improvement of the photocathode sensitivity}

A photocathode is one of the most important parts of PMTs as a photon sensor to translate photons to electrons. Photons are absorbed in a photocathode to excite electrons. The electrons travel to the boundary and emitted to vacuum. In general, thicker photocathode absorbs photons more efficiently, however, probability of recombination for exited electrons increases rapidly before reaching the boundary. Therefore, there is optimized thickness and composition of materials for high-sensitive photocathode. Figure 3 shows spectral sensitivities of conventional photocathodes. In general, a bialkali photocathode is used to detect $400 \mathrm{~nm}$ photons, whereas a multialkali or extended red one for 550 to $800 \mathrm{~nm}$. As shown here, the maximum quantum efficiency is 20 to $30 \%$, in spite of such optimization.

Recently, one of our optimization methods has succeeded to develop a photocathode of higher quantum efficiency. Figure 4 shows spectral responses of such photocathodes called super bialkali (SBA) and ultra bialkali (UBA). In the case of R7400 series, the maximum quantum efficiency made a big jump from $27 \%$ to $46 \%$ with the UBA. At this moment, 
application of SBA or UBA to the other PMTs is limited, because optimization depends on detailed structure of PMTs. Hamamatsu is going to expand the availability of these photocathodes for variety of PMTs.

For the higher quantum efficiency in green or red region, semiconductor crystal photocathode is useful. Due to good quality of these crystals, life time of exited electrons is much longer than that in alkali-based photocathode. Thus, a photocathode as thick as $1 \mu \mathrm{m}$ can be used to absorb more photons. As GaAs or GaAsP crystals show negative electron affinity when they are activated by cesium, the probability of photo-electron emission is high. As a result, quantum efficiencies of these photocathodes are high, as shown in Figure 5. The maximum quantum efficiency of GaAsP photocathode reaches $50 \%$ at visible region. The availability of these photocathodes is very limited, because different activation process from conventional photocathodes is necessary. At this moment, R7422 series with $5 \mathrm{~mm}$ diameter [3], or R10467 series (HPD) with $3 \mathrm{~mm}$ diameter are the only choice.



Figure 3: Quantum efficiencies of conventional photocathodes, such as bialkali, multialkali, and extended red multialkali are shown.

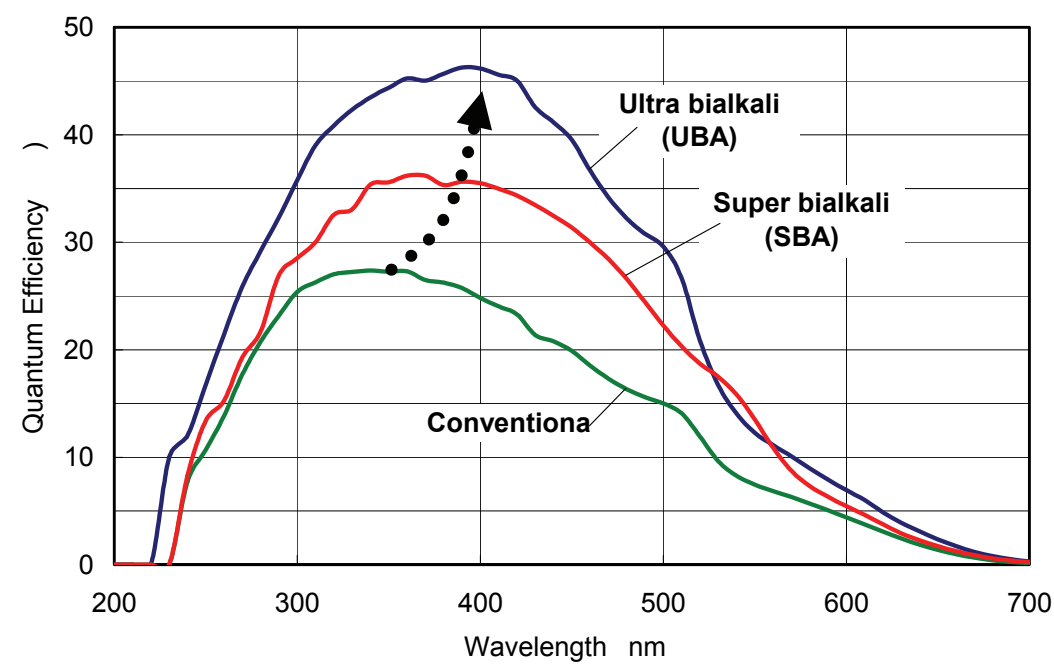

Figure 4: Quantum efficiencies of new bialkali photocathodes called super bialkali (SBA) and ultra bialkali (UBA) are shown, referring to R7400 series. Big jump of $27 \%$ to $46 \%$ at the maximum quantum efficiency is shown. 


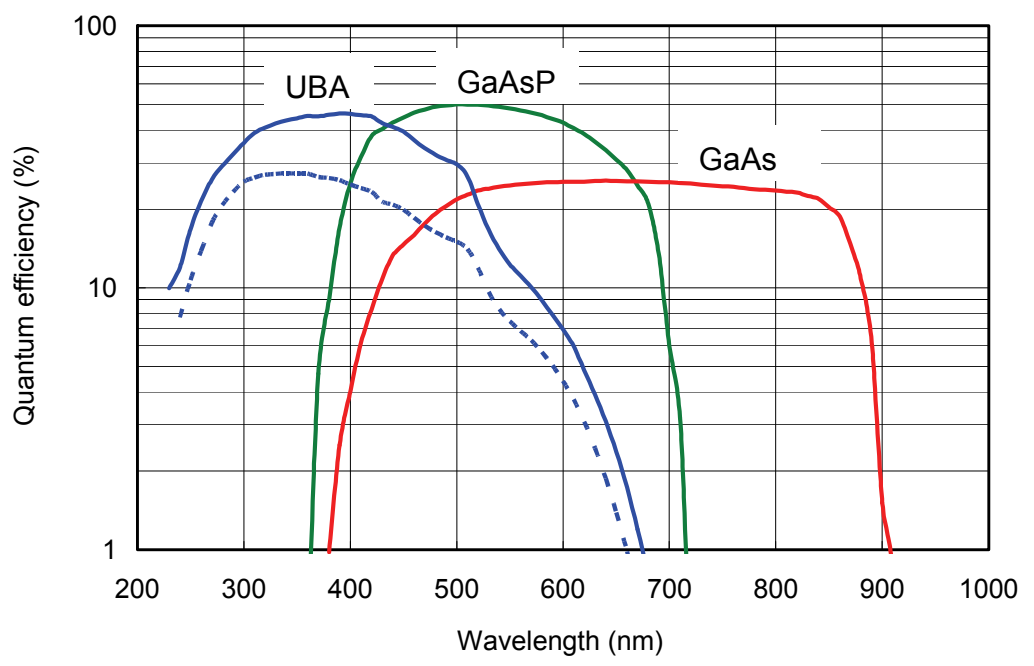

Figure 5: Quantum efficiencies of semiconductor crystal photocathodes are shown with UBA. The maximum quantum efficiency of GaAsP photocathode reaches $50 \%$ in visible region.

\subsection{Metal channel dynodes}

In order to realize small PMT, a dynode chain made of thin etched plates was developed. Etched parts of the plates are finely assembled to establish a bunch of individual dynodes as shown in Figure 6 [4]. This looks like a channel of electrons, and is called as a metal channel dynode. Thanks to this structure, PMT with $12 \mathrm{~mm}$-length (R7400 series) are realized. Because of short electron trajectory, R7400 performs fast rise and fall times of the order of nano second. In addition, multi-pixel or position sensitive sensors are available, such as R7600 series, because incident position of electrons is preserved in the metal channel dynode.

The metal channel dynode was further investigated to develop so called flat panel PMT, as shown in Figure 7. Large effective area of $49 \mathrm{~mm}$ square compared with the outer dimension of $52 \mathrm{~mm}$ is one of the features of this PMT. Models of 8x8 pixels (H8500) and 16x16 pixels (H9500) [5] are available for applications which requires fine segmentations such as highenergy physics experiments and PET.

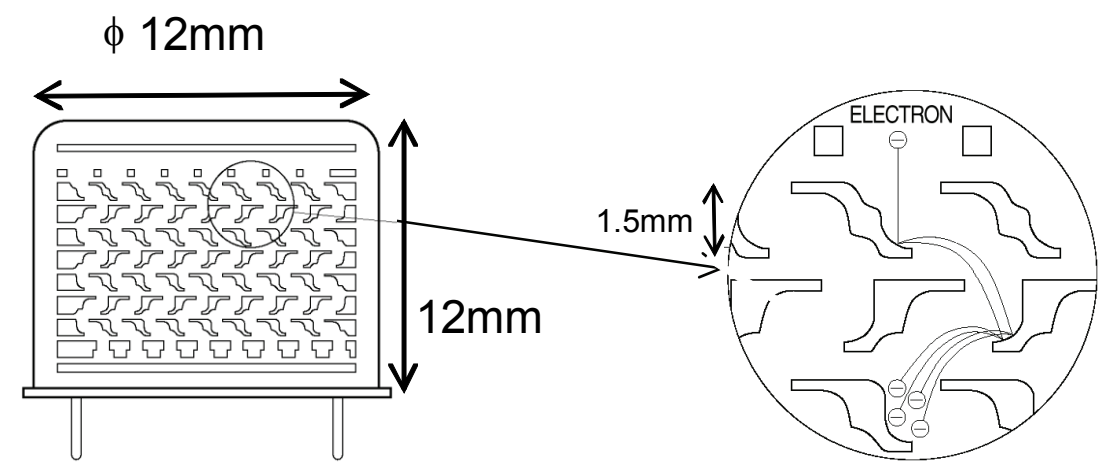

Figure 6: Sectional drawing of R7400 series shows detailed structure and operation of metal channel dynode. 


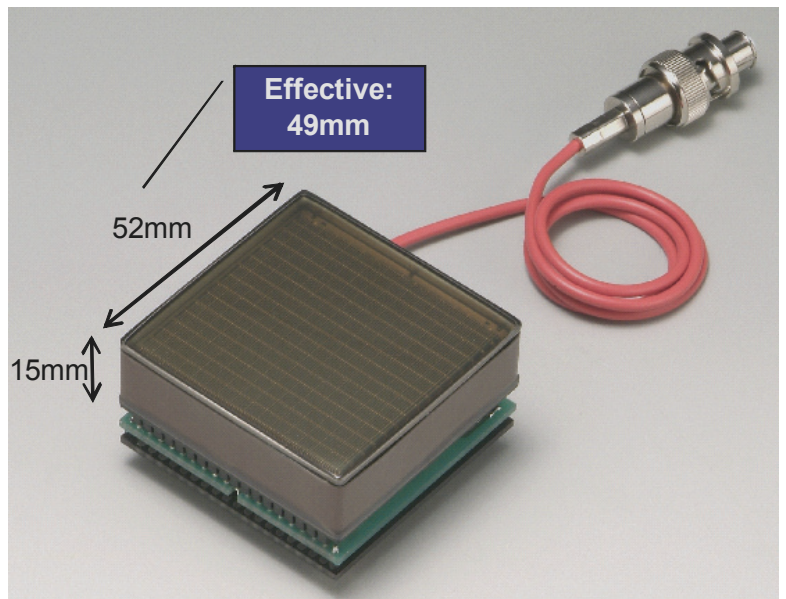

Figure 7: Photograph of flat panel PMT (H8500/H9500).

\section{$2.4 \quad$ MCP}

A mcirochannel plate (MCP) is a thin glass plate with plenty of via holes from input to output surfaces. Typical diameter of holes is $6 \mu \mathrm{m}$, and thickness is $240 \mu \mathrm{m}$, thus the aspect ratio of hole is 40 . With a bias voltage of $1 \mathrm{kV}$, for example, between input and output surfaces, an electron entering a hole is multiplied by successive secondary electron multiplication to be a factor of 10,000, as shown in Figure 8 [6].

Two layers of MCP can be used for dynodes to realize so called MCP-PMT. Two MCPs give enough high gain of $10^{6}$ to detect single photons. Because of very short electron trajectory in a channel of the MCP, the MCP-PMT shows good timing resolution of less than $30 \mathrm{ps}$ in FWHM for single photons [7]. With the same reason, it is insensitive to magnetic field of up to $1.8 \mathrm{~T}$, when the field is parallel to the tube axis [7]. Due to these great features, MCP-PMT is studied to be used for TOP counters in Belle upgrade [8]. One of the drawbacks of MCP is an open area ratio of $60 \%$, approximately, thus $40 \%$ of incident electrons are not multiplied efficiently.
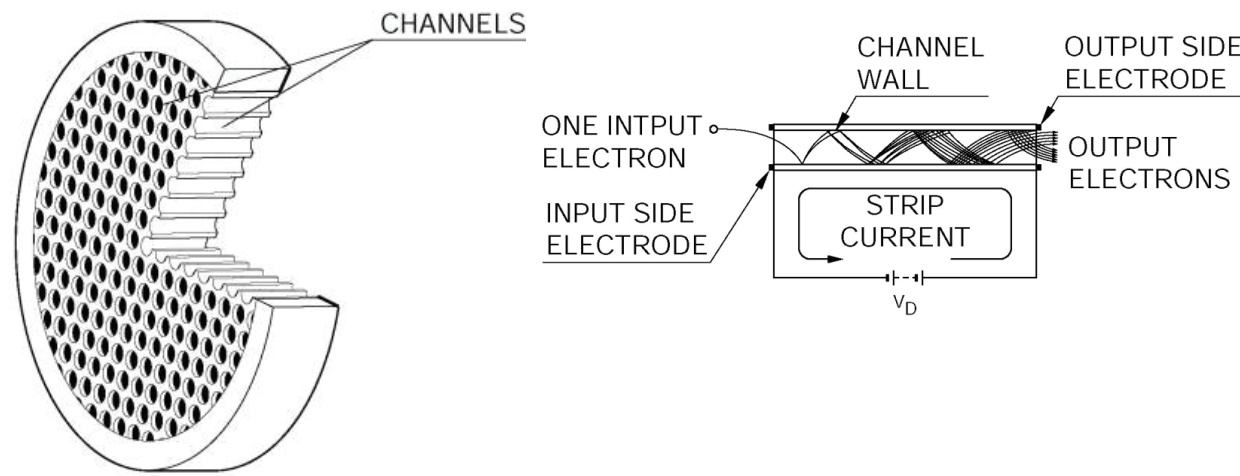

Figure 8: Sectional drawing of MCP shows the way for electrons being multiplied in a channel. 


\section{Hybrid Photo-Detector (HPD)}

When electrons deposit their kinetic energy in a silicon device, one electron-hole pair is generated per $3.6 \mathrm{eV}$ in average. This process can be used for multiplying the photo electrons instead of a dynode chain. The photon sensor incorporating a semiconductor device facing the photocathode is called a hybrid photo-detector (HPD). Performance of a high-speed HPD developed recently, as well as general features of the HPD, is reported in this section.

\subsection{Structure and common features of HPD}

Typical structure of HPD is schematically shown in Figure 9. Electrons emitted from the photocathode in response to incident photons are accelerated and focused on the avalanche diode (AD) by an electron lens. Electrons deposit their kinetic energy in the AD, and generate electron-hole pairs. Number of electron-hole pairs is linearly proportional to landing energy of electrons, except energy lower then $2 \mathrm{keV}$, and is typically 1,500 to 2,000 at $8 \mathrm{keV}$. These multiplied electrons are drift to the avalanche region by an electric field in the $\mathrm{AD}$ to be further multiplied by a factor of 100 . Then, the total gain reaches $10^{5}$, high enough to detect single photons. Non linear part at the low energy part can be explained by rapid recombination of carriers at surface states, because carriers are generated close to the surface (order of nm).

The advantage of the HPD compared with PMTs is a factor of 100 higher gain at the first gain stage; electron-bombarded gain. This makes almost noiseless multiplication of electrons, and gives a capability to distinguish number of electrons from the photocathode. Simple structure of HPD has a potential to be high speed, as shown below.

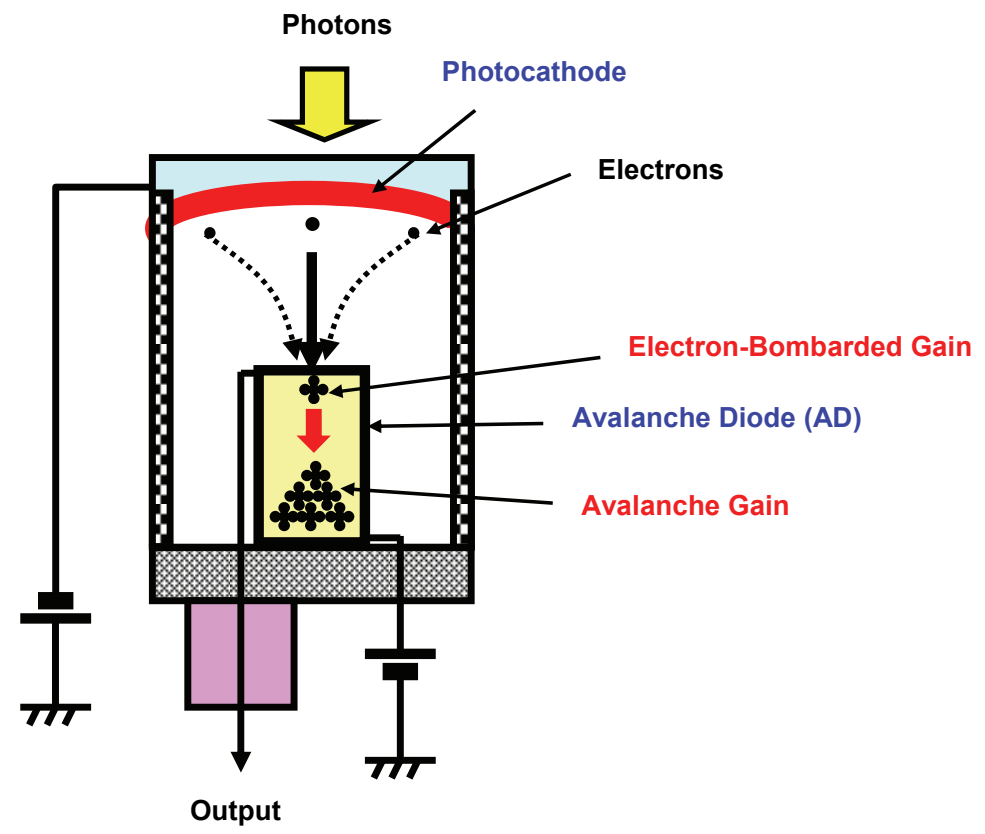

Figure 9: Schematic drawing of HPD shows that electrons entering the AD generate thousands of electron-hole pairs at the first point, and these electrons are further multiplied by avalanche multiplication. 


\subsection{High-speed HPD}

Adjusting transit time from the photocathode to the $\mathrm{AD}$, and using small $\mathrm{AD}$ for fast response, the high-speed HPD was designed and manufactured [9]. A bialkali photocathode of 8 $\mathrm{mm}$ in diameter is fabricated on the round-shaped faceplate. Electrons from the photocathode are focused on the $\mathrm{AD}$ of $1 \mathrm{~mm}$ in diameter.

The gain characteristics of both electron-bombardment and avalanche multiplication are shown in figures 10 and 11 , respectively. Total gain reaches 180,000 , where the electronbombarded gain is 1600 at the photocathode voltage of $-8 \mathrm{kV}$, and the avalanche gain is 110 at $405 \mathrm{~V}$ to the $\mathrm{AD}$. The rise and fall times for short pulsed light are 360 and $340 \mathrm{ps,}$, respectively, as shown in Figure 12. Pulse height spectrum for several photons indicates capability to distinguish up to 6 electrons from the photocathode, as shown in Figure 13. The timing resolution for single photons was measured to be 50 ps in FWHM for full illumination, and 33 ps for illumination of central area of $1 \mathrm{~mm}$ diameter. This apparently indicates imperfection of the adjustment of transit time over entire photocathode, however, timing resolution of $50 \mathrm{ps}$ is better than the fastest PMT of 200 ps, approximately.

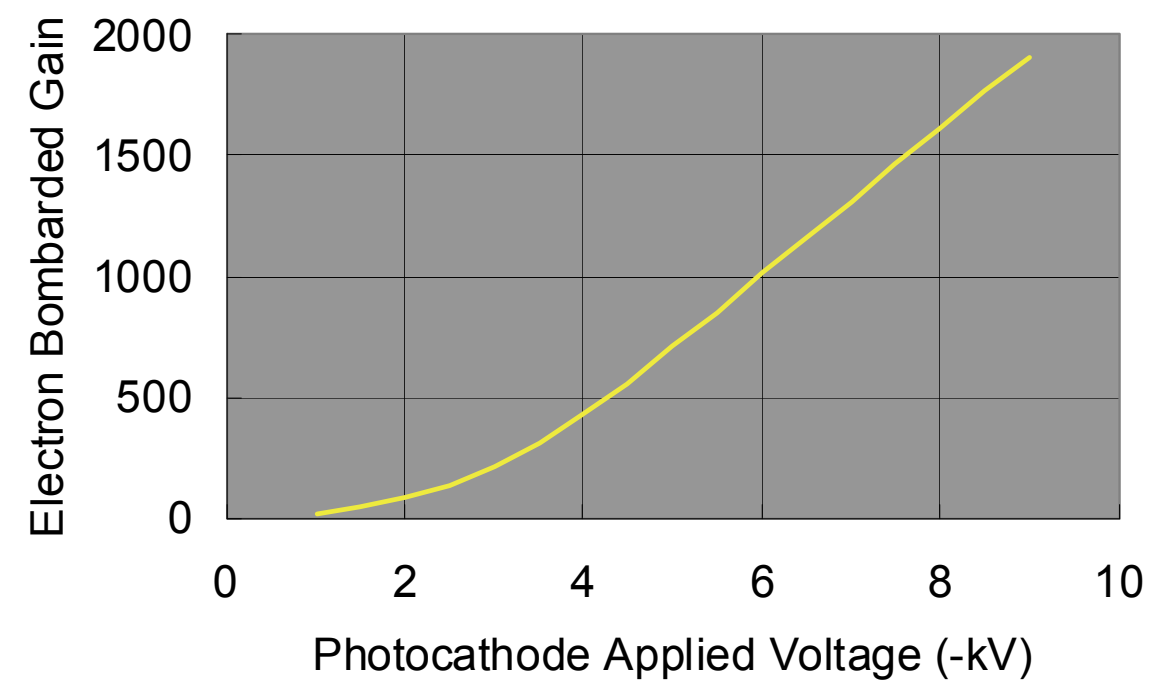

Figure 10: Electron-bombarded gain characteristic shows gain of 1600 at the photocathode voltage of $8 \mathrm{kV}$. 


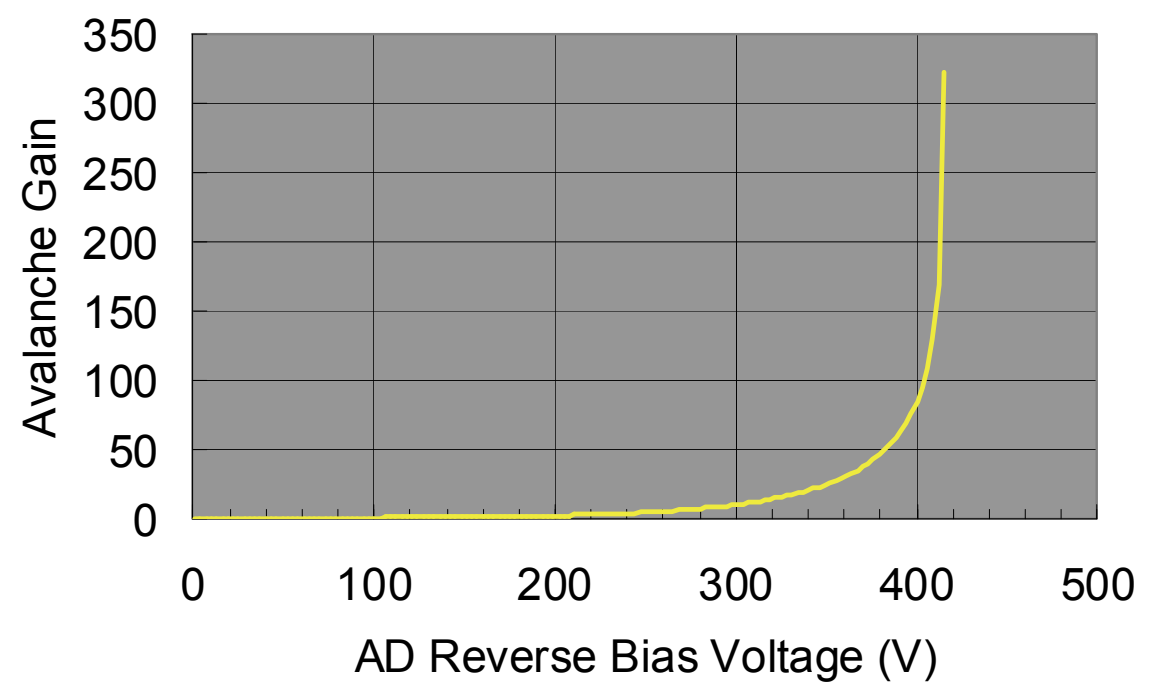

Figure 11: Avalanche gain characteristics as a function of the bias voltage to the AD.

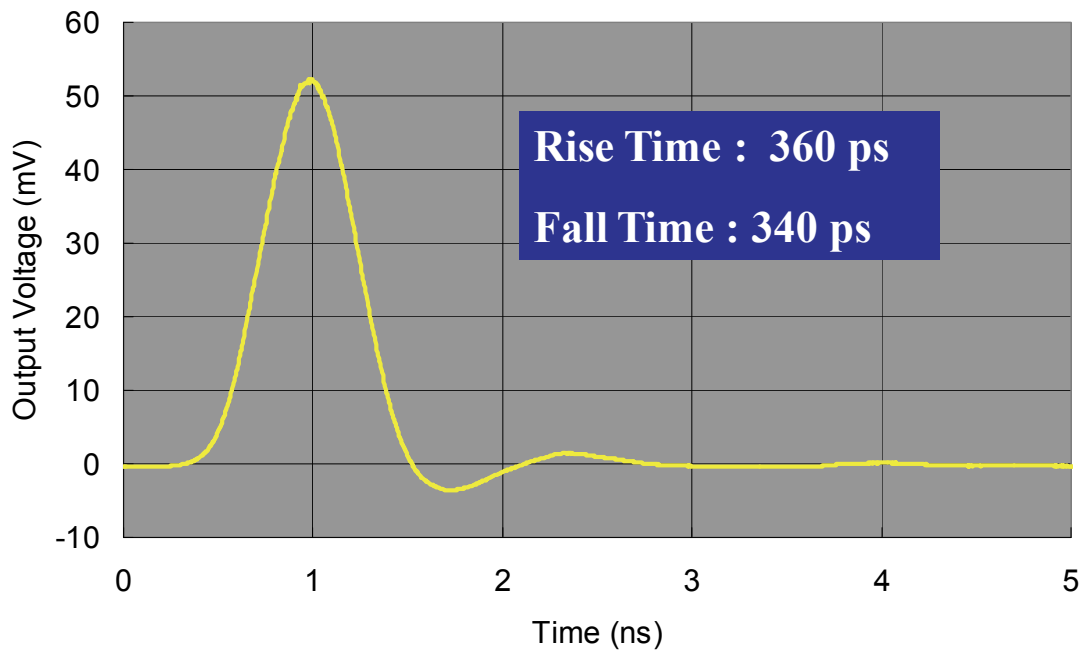

Figure 12: Output waveform for impulse-light input. 




Figure 13: The pulse height spectrum for multi photons clearly shows peaks corresponding up to 6 photoelectrons.

\subsection{HPD under development}

Several HPDs are under development now. One of them has a GaAsP photocathode of 18 $\mathrm{mm}$ in diameter for MAGIC telescope [10]. The other has a bialkali photocathode of $60 \times 60 \mathrm{~mm}$ facing to the $\mathrm{AD}$ of $12 \times 12$ pixel, being developed for aerogel RICH counters. Current status is shown in ref [11]. Regarding multi-pixel HPD, 8x8 pixel type was reported in [12]. The last one has a large format photocathode of 13 inches being developed for water Cherenkov counter of the next generation [13].

\section{Summary}

In spite of long history of 50 years, PMTs are still evolving continuously satisfying the requirements from all science frontiers. Some of the recent advancements are shown above. HPD, a new type of vacuum device to detect single photons, appeals its capability, and rapidly expands its application these days.

\section{References}

[1] Catalogue of Hamamatsu photonics K.K., PhotomultiplierTtubes, 2006.

[2] K. Kinoshita et al., Femtosecond streak tube, Rev.Sci. Instrum.," 58 (6), pp. 932-938, 1987.

[3] Catalogue of Hamamatsu photonics K.K., PHOTOMULTIPLIER TUBE MODULES, 2007.

[4] Catalogue of Hamamatsu photonics K.K., R7400U SERIES, 2004.

[5] Catalogue of Hamamatsu photonics K.K., H9500, 2006.

[6] Catalogue of Hamamatsu photonics K.K., MCP \& MCP assembly, 2007. 
[7] Technical handook of Hamamatsu photonics K.K., PHOTOMULTIPLIER TUBES, Third edition, 2005.

[8] K. Inami, Timing properties of MCP-PMT, International workshop on new photon-detectors PD07 (this workshop).

[9] A. Fukasawa et al., High-speed HPD for photon counting, Conference record of IEEE Nuclear Science Symposium, 2006.

[10] T. Saito, Favourable properties of HPD R9792U-40 for the MAGIC telescope project, International workshop on new photon-detectors PD07 (this workshop).

[11] I. Adachi, Development of 144 multi-anode HPD for Belle aerogel RICH photon detector, International workshop on new photon-detectors PD07 (this workshop).

[12] M. Suyama et al., Development of a Multipixel Hybrid Photodetector With High Quantum Efficiency and Gain, IEEE Trans. Nucl. Sci., vol. 51, No.3, pp. 1056-1059, 2004.

[13] Y. Kawai et al., Large-aperture hybrid photo-detector, Nucl. Instrum. Methods A579, pp.42-45, 2007. 